\title{
Two cases of long-lasting, sub-microscopic Plasmodium malariae infections in adults from coastal Tanzania
}

\author{
Tobias Schindler ${ }^{1,2^{*}} \mathbb{D}$, Said Jongo ${ }^{3 *}$, Fabian Studer ${ }^{1,2}$, Maximilian Mpina $^{1,2,3}$, Grace Mwangoka ${ }^{3}$, \\ Sarah Mswata ${ }^{3}$, Kamaka Ramadhani ${ }^{3}$, Julian Sax ${ }^{1,2}$, L. W. Preston Church ${ }^{4}$, Thomas L. Richie ${ }^{4}$, Marcel Tanner ${ }^{1,2}$, \\ Stephen L. Hoffman ${ }^{4}$, Salim Abdulla ${ }^{3}$ and Claudia Daubenberger ${ }^{1,2}$
}

\begin{abstract}
Background: Malaria is endemic in Tanzania with majority of clinical cases caused by Plasmodium falciparum. Additionally, Plasmodium malariae and Plasmodium ovale spp. are also present and clinical manifestations caused by these infections are not well described. Clinical episodes caused by P. malariae infections are often characterized by a relatively mild illness with a low number of parasites, which can persist for long periods. In this report, two cases of P. malariae infections that were identified during a clinical trial evaluating the P. falciparum malaria vaccine candidate, PfSPZ Vaccine are described. The two participants were followed up and monitored for clinical and laboratory parameters to assess vaccine safety providing the opportunity to study clinical manifestations of $P$. malariae over 4 months.

Case presentation: Two young, healthy Tanzanian men infected with low density asexual blood stage P. malariae diagnosed by quantitative polymerase chain reaction (qPCR) are described. Retrospective analysis of collected and stored blood samples revealed that the two volunteers had constant asexual blood stage parasitaemia for more than 4 months. During the 132 days of infection, the volunteers' vital signs, body temperature and serum biochemistry all remained within normal ranges. Haematological abnormalities, which were transiently outside normal ranges, were regarded as not clinically significant. During this time period, four consecutive evaluations of blood samples by thick blood smear microscopy conducted by an experienced microscopist were all negative, indicating the presence of low-density sub-microscopic infections.
\end{abstract}

Conclusions: The two cases of $P$. malariae infections presented here confirm the ability of this Plasmodium species to persist at low density in the human host for extended time periods without causing clinical symptoms. The presented data also demonstrate that clinical study sites in malaria endemic regions need to have a strong malaria diagnostic infrastructure, including the ability of capturing sub-microscopic parasitaemia and differentiation of Plasmodium species.

Trial registration ClinicalTrials.gov: NCT02613520, https://clinicaltrials.gov/ct2/show/NCT02613520, Registered: November 24th 2015, Enrolment of the first participant to the trial: December 15th 2015, Trial was registered before the first participant was enrolled

Keywords: Plasmodium malariae, Asymptomatic malaria, Quantitative polymerase chain reaction (qPCR)

\footnotetext{
*Correspondence: tobias.schindler@unibas.ch; sjongo@ihi.or.tz

${ }^{1}$ Department of Medical Parasitology and Infection Biology, Swiss

Tropical and Public Health Institute, Basel, Switzerland

${ }^{3}$ Ifakara Health Institute, Bagamoyo Research and Training Centre,

Bagamoyo, United Republic of Tanzania

Full list of author information is available at the end of the article
} 


\section{Background}

Malaria is endemic in Tanzania with more than $90 \%$ of the population at risk and 5.6 million cases reported by the public health sector in 2017 [1]. While Plasmodium falciparum is the dominant malaria species responsible for majority of infections and deaths, other Plasmodium species are also endemic in Tanzania. David Clyde, who served as the director of the Malaria Service of the East African Malaria Institute at Amani, described the occurrence of four human malaria species, including $P$. falciparum, Plasmodium vivax, Plasmodium ovale spp. and Plasmodium malariae in his 1967 book "Malaria in Tanzania" [2]. Plasmodium vivax was attributed to importation by Indian immigrants during the first world war and since 1917 this influx has virtually ceased. Plasmodium malariae was observed in 10-20\% of malaria infections, mainly as co-infections with $P$. falciparum and during childhood [2]. More recently, a microscopy-based crosssectional survey conducted in the Tanga region of coastal Tanzania found very few infections with $P$. malariae (0.3\%) or P. ovale spp. (0.1\%) [3]. Data collected in coastal Tanzania, confirm these low numbers of non-P. falciparum Plasmodium infections. Diagnosis by qPCR revealed low prevalence for P. malariae $(0.7-5.8 \%)$ and $P$ ovale spp. (0.9-1.1\%) among asymptomatic school children (Schindler et al., unpublished data). Since microscopic diagnosis of $P$. malariae asexual blood stage parasites is hampered by the low parasitaemia and morphological similarities to $P$. falciparum, molecular based, highly sensitive diagnostic methods are needed to establish the true prevalence of this parasite in the population [4]. Lack of sensitive $P$. malariae diagnosis methods applicable in the field and the research focus on $P$. falciparum has resulted in significant knowledge gaps regarding spectrum of potential clinical manifestations and burden of $P$. malariae infections [5].

It is well established that $P$. malariae is widespread throughout sub-Saharan Africa, South East Asia and Latin America and the biology of $P$. malariae was reviewed by Collins et al. [6]. Treatment of syphilis by controlled infections with $P$. malariae provided valuable insight into human-parasite interactions. The red blood cell cycle lasts $72 \mathrm{~h}$ with an average of 8 merozoites released per schizont and the parasite prefers to infect and develop in older erythrocytes. So far, no evidence for a dormant liver stage as described in $P$. vivax and $P$. ovale spp. has been observed. Faster acquisition of immunity against $P$. malariae compared to immune responses against $P$. falciparum has been described [6].

Clinical episodes of $P$. malariae infections are characterized by a mild illness caused by low numbers of parasites which can persist for extremely long periods, often for years or even decades [6]. There are reports of cases of P. malariae caused illness from Greece [7] and Trinidad and Tobago [8] decades after eradication of malaria from these regions. Chronic $P$. malariae infections have been considered a major cause of the nephrotic syndrome in the past, although the incidence of $P$. malariaassociated nephrotic syndrome has been dramatically reduced in recent decades [7-9]. Recently, it was demonstrated that the controlled infection of two volunteers with cryopreserved $P$. malariae-infected erythrocytes was well tolerated and no severe or serious adverse effect, or biochemical abnormalities were observed [10].

The clinical research facility of the Ifakara Health Institute in Bagamoyo, Tanzania, conducts clinical trials evaluating efficacy of experimental malaria vaccines in the target population [11-13]. A controlled human malaria infection (CHMI) model has been successfully established since 2012 [14]. As part of these clinical trials, participants are closely monitored to identify any abnormal clinical or laboratory parameters in order to evaluate vaccine safety and tolerability. Regularly, volunteers are screened for Plasmodium spp. parasites in blood using thick blood smear microscopy as well as quantitative polymerase chain reaction (qPCR). The volunteers described in this report participated in a study evaluating the safety and efficacy of immunization with Sanaria ${ }^{\circledR}$ PfSPZ Vaccine composed of radiation attenuated, aseptic, purified, cryopreserved P. falciparum sporozoites (PfSPZ) [11, 15-19] which was conducted between 2015 and 2016 (clinicaltrials.gov: NCT02613520) [20]. The clinical cases of two young men infected with asexual blood stage $P$. malariae as diagnosed by $\mathrm{qPCR}$ are described. These volunteers were followed closely for 4 months during the clinical trial.

\section{Case presentation}

Two male residents of Bagamoyo, 20 and 22 years of age, were enrolled into the clinical trial based on predefined exclusion and inclusion criteria as outlined in the clinical trial protocol. A review of the medical history, physical examination, vital signs (pulse, blood pressure, and respiratory rate), and ECG did not reveal any abnormalities. At screening, the volunteers had negative serologies for human immunodeficiency virus (HIV), hepatitis B virus (HBV), and hepatitis $\mathrm{C}$ virus ( $\mathrm{HCV})$. A single stool sample collected at study enrolment was negative for intestinal helminths and no Schistosoma haematobium eggs were detected in urine. No blood biochemistry abnormalities were detected, which included alanine aminotransferase (ALT), aspartate aminotransferase (AST), total bilirubin (BIL), and creatinine (CRE). The urine analysis using a 13-parameter dipstick (Combina 13 test strips, HUMAN Diagnostics, Germany) was negative at enrolment. A complete blood count (CBC) was 


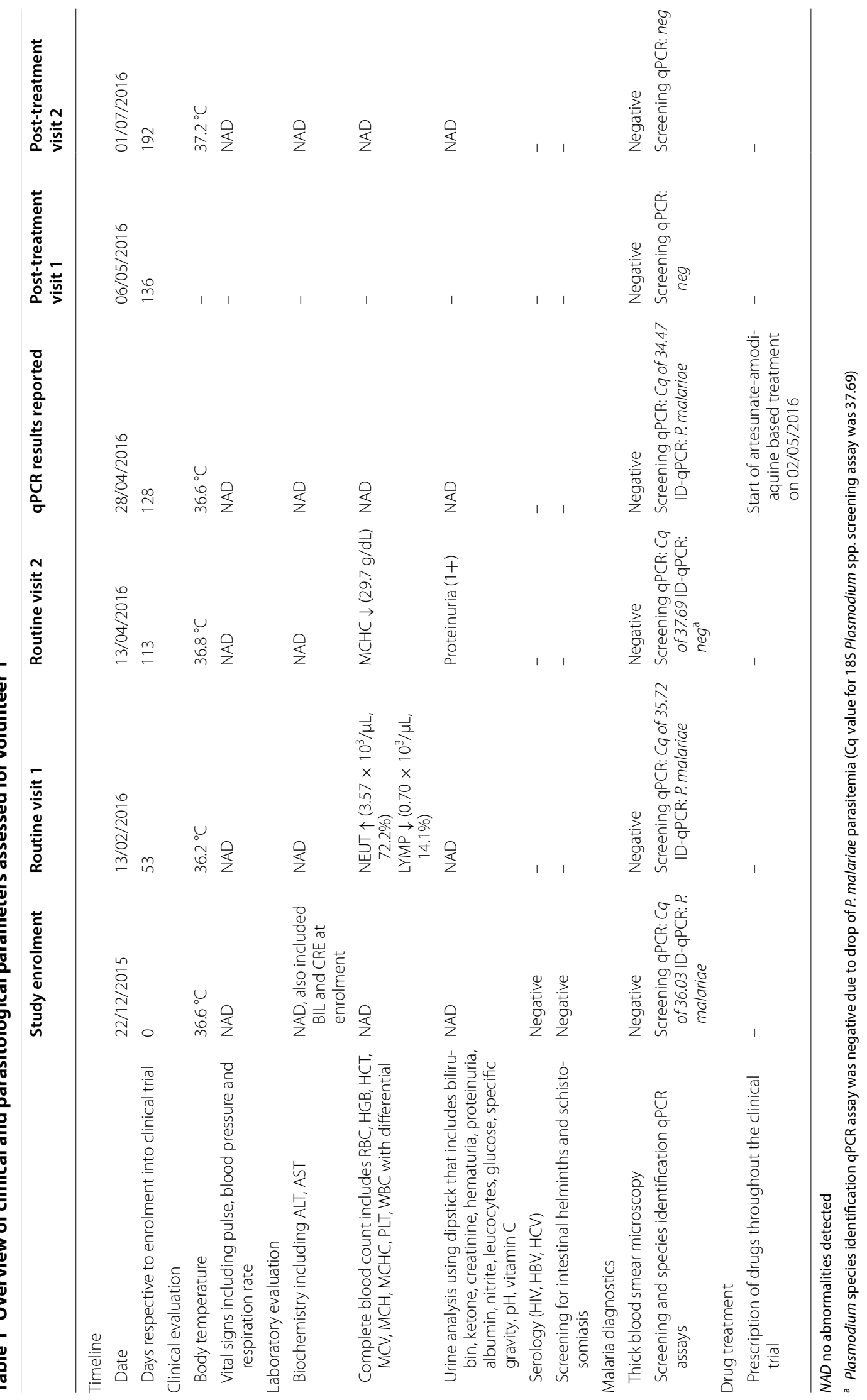




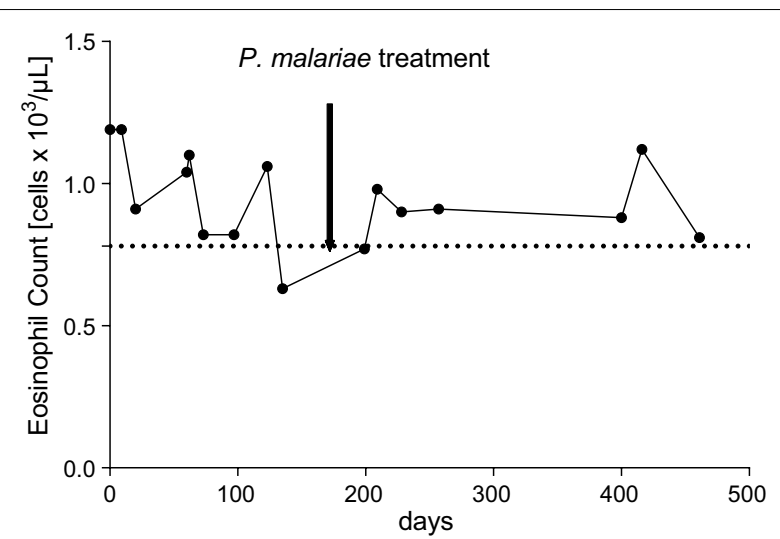

Fig. 1 Elevated eosinophil counts for volunteer 2 over a time period of more than 400 days. Eosinophil counts of volunteer 2 covering all visits, from study enrolment to completion, are shown. The dashed line represents the upper limit of the normal range $\left(0.78 \times 10^{3}\right.$ cells/ $\mu \mathrm{L})$ conducted at screening and showed normal haematological parameters for volunteer 1 , while volunteer 2 had elevated eosinophil counts $\left(1.19 \times 10^{3} / \mu \mathrm{L}\right.$ compared to the pre-defined upper normal range of $0.78 \times 10^{3}$ cells $\left./ \mu \mathrm{L}\right)$. During the 16 weeks of immunization (3 doses of PfSPZ Vaccine at 8-week intervals), the volunteers' health status was closely monitored. Every 8 weeks at pre-defined visits blood was drawn and any deviations from reference laboratory values were reported. Vital signs, body temperature and biochemistry remained within normal ranges when assessed four times during the follow up period. Haematological parameters which were transiently outside normal ranges in both volunteers were regarded as not clinically significant (Tables 1,2 ). Noteworthy, for volunteer 2 the eosinophil counts remained consistently elevated until the end of the study (Fig. 1). Both volunteers tested once positive for low grade proteinuria, during the study by urine dipstick (Tables 1,2 ).

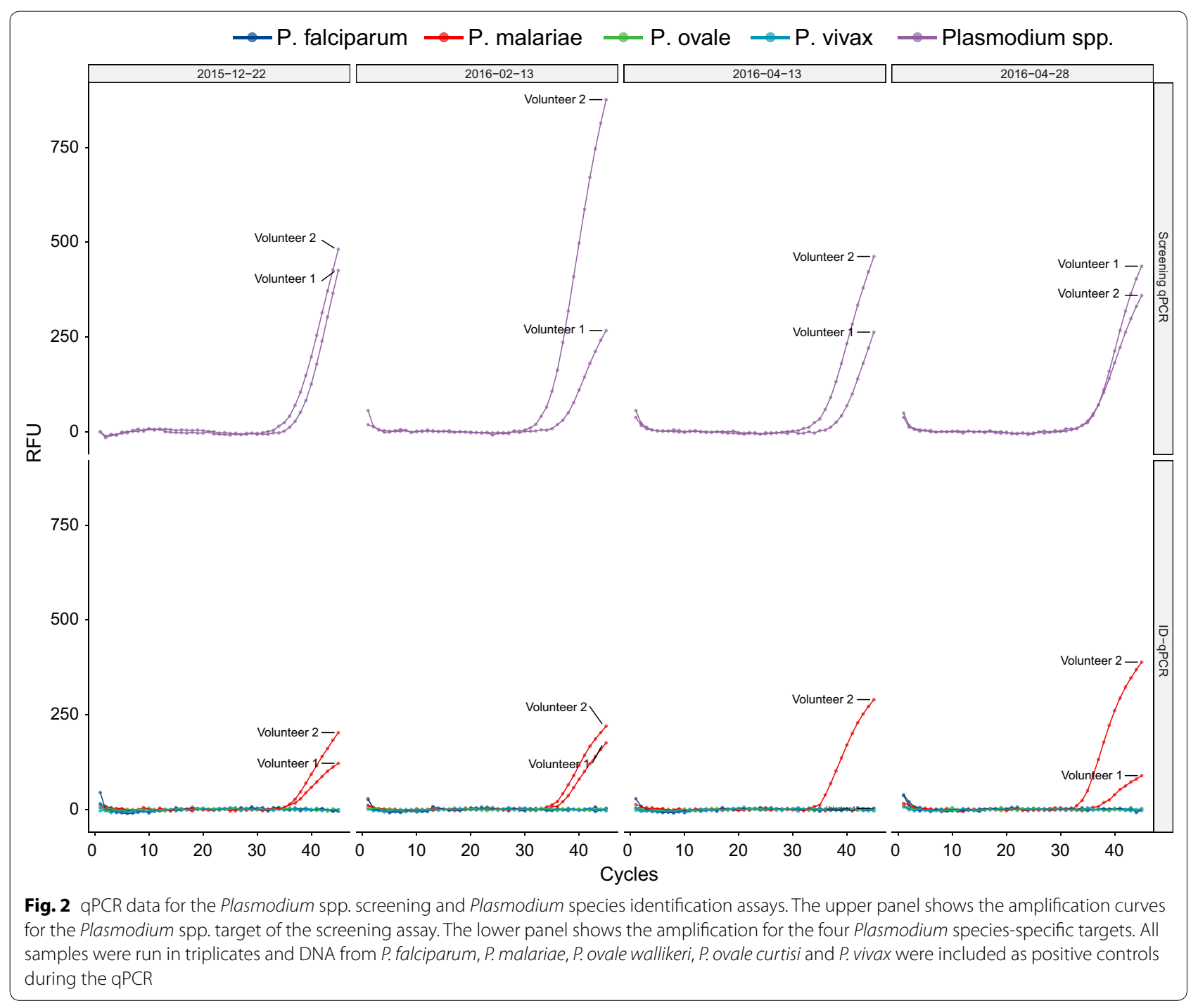


After concluding three PfSPZ Vaccine immunizations, and before vaccine efficacy was assessed by CHMI, the study protocol required screening of whole blood by qPCR to detect sub-microscopic malaria parasitaemia, so that these volunteers could be treated accordingly before participation in CHMI. During this routine visit, qPCR was conducted with fresh blood samples and it was discovered that two volunteers were infected with Plasmodium spp. parasites [21]. Based on P. malariae species-specific qPCR [22] and conventional nested PCR [23], P. malariae infections were confirmed. The presence of P. falciparum [24, 25], P. ovale spp. [26], and P. vivax [25] was excluded by qPCR and conventional PCR [23].

Treatment with 3 doses of artesunate/amodiaquine (200/540 mg) daily for 3 days was initiated, and complete parasite clearance was confirmed by qPCR 4 days later. Both volunteers then underwent $\mathrm{CHMI}$ and remained in the clinical trial until study completion. Within the following 296 days until the completion of the clinical trial, no recurrent (recrudescence or new infection) P. malariae parasitaemia was observed. Both volunteers were negative for $P$. falciparum after the first CHMI, and both became positive for P. falciparum after a second CHMI at 40 weeks after the last immunization and were successfully treated with artemether/lumefantrine.

Blood samples collected during the clinical trial and stored frozen were analysed retrospectively by $\mathrm{qPCR}$ to determine the time point of $P$. malariae infection. It turned out that both volunteers had $P$. malariae parasitaemia at enrolment into the clinical trial. Both volunteers remained positive throughout the vaccination period. Plasmodium malariae parasites were detectable at four out of four clinical visits, namely at day 0,53 , 113 and 128 of study and malaria treatment took place 132 days after the first detection of the $P$. malariae infections (Fig. 2). Evaluation of four blood samples collected at the same days by thick blood smear microscopy and conducted by an experienced microscopist was reported as negative. Thick blood smear preparation and reading was performed according to our standard operating procedure followed during CHMI studies [14]. The negative microscopy results and the high $\mathrm{Cq}$ values (median of 34.1 with a range of 31.6-37.7) obtained by the Plasmodium spp. qPCR assay indicate that the parasitaemia levels were low. When compared to qPCR based detection of $P$. falciparum $18 \mathrm{~S}$ gene, these Cq values would correspond to a parasitaemia between 1 and $10 P$. malariae parasites per $\mu \mathrm{L}$ blood [27].

\section{Discussion and conclusion}

The two $P$. malariae cases presented here confirm the ability of this Plasmodium species to persist at low density in the human host for extended time periods without causing clinical symptoms or signs. Both were detected in clinically healthy, young men participating in a clinical trial of PfSPZ Vaccine. No abnormalities in vital signs, alanine aminotransferase, aspartate aminotransferase, total bilirubin, and creatinine serum levels were detected. Except for a one-time low-level proteinuria, urine analysis parameters measured by dipstick remained within physiological ranges and there was no indication of impaired renal function in these two volunteers. Volunteer 2 did have mildly elevated eosinophil counts throughout the entire course of the clinical trial. These levels were not affected by the treatment of the P. malariae infection and may have reflected an ongoing intestinal helminth infection that was too low to be detected by a single stool examination. All other haematological abnormalities were of temporary nature and considered to be not clinically significant. Interestingly, the $P$. malariae parasitaemia levels were not affected by the three rounds of PfSPZ Vaccine immunizations. This might be due the mode-of-action of the vaccine which is thought to act against the liver-stage of the parasite.

The data presented in this report demonstrates that study sites in malaria endemic regions conducting clinical trials should develop on site malaria diagnostic infrastructure, which includes the detection of low-density asexual blood stage parasitaemia and identification of different Plasmodium species. Eventually, if the goal of malaria elimination is pursued vigorously, the implementation of highly sensitive diagnostic methods to detect asymptomatic, low-density $P$. malariae infections need to be included into the malaria elimination agenda.

\section{Abbreviations}

PfSPZ: P. falciparum sporozoites; CHMl: controlled human malaria infections; HIV: human immunodeficiency virus; HBV: hepatitis B virus; HCV: hepatitis C virus; ALT: alanine aminotransferase; AST: aspartate aminotransferase; BIL: total bilirubin; CRE: creatinine; CBC: complete blood count; RBC: red blood cells; HGB: haemoglobin; HCT: haematocrit; MCV: mean corpuscular volume; MCH: mean corpuscular haemoglobin; MCHC: mean corpuscular haemoglobin concentration; PLT: platelets; WBC: white blood cells; NEUT: neutrophils; LYMP: lymphocytes; EO: eosinophils; RDW-SD: red cell distribution width; TBS: thick blood smear; qPCR: quantitative polymerase chain reaction; Cq: quantification cycles of qPCR reaction; ID-qPCR: Plasmodium species identification qPCR assay; NAD: no abnormalities detected.

\section{Authors' contributions}

SJ, MT, SLH, SA and CD designed the study. TS, MM, GM, SM and JS conducted the laboratory analysis. SJ, FS, KR, LWPC and TLR collected and analysed the clinical data. TS, SJ, SLH, LWPC, TLR and CD interpreted the findings and wrote the manuscript. All authors read and approved the final manuscript.

\section{Author details}

${ }^{1}$ Department of Medical Parasitology and Infection Biology, Swiss Tropical and Public Health Institute, Basel, Switzerland. ${ }^{2}$ University of Basel, Basel,

Switzerland. ${ }^{3}$ Ifakara Health Institute, Bagamoyo Research and Training Centre, Bagamoyo, United Republic of Tanzania. ${ }^{4}$ Sanaria Inc., Rockville, MD, USA. 


\section{Acknowledgements}

The authors would like to thank the study volunteers for their participation in the study. We would also like to thank Paul Lansdell, PHE Malaria Reference Laboratory and Don van Schalkwyk, London School of Hygiene and Tropical Medicine who kindly provided the DNA controls for the different Plasmodium species.

\section{Competing interests}

LWP Church, TL Richie and SL Hoffman are salaried, full time employees of Sanaria Inc, the developer and sponsor of Sanaria ${ }^{\circledR}$ PfSPZ Vaccine. These individuals were not responsible for the collection, recording or entry of the clinical and parasitological data presented here. The other authors have no relevant affiliations or financial involvement with any organization or entity with a financial interest in the subject matter or materials discussed in the manuscript.

\section{Availability of data and materials}

The datasets supporting the conclusions of this article are included within the article.

\section{Consent for publication}

Written informed consent was granted from the patients for publication of clinical and laboratory data obtained during the clinical trial on condition of anonymity.

\section{Ethics approval and consent to participate}

The two volunteers described in this case report consented to participate in a clinical study, registered at Clinical Trials.gov (NCT02613520), and conducted under a U.S. FDA IND application. The study was performed in accordance with Good Clinical Practices. All data presented in this case report were obtained according to the approved study protocol. The protocol was approved by the institutional review boards of the Ifakara Health Institute (Ref. No. IHI/IRB/129 No: 32-2015), and the National Institute for Medical Research Tanzania (NIMR/HQ/R.8a/Vol.IX/2049), by the Ethikkommission Nordwest- und Zentralschweiz, Basel, Switzerland (reference number 15/104), and by the Tanzania Food and Drug Authority (Auth. No. TZ15CT013).

\section{Funding}

This study was funded by the public-private partnership, the Equatorial Guinea Malaria Vaccine Initiative (EGMVI), supported by the Government of Equatorial Guinea - Ministry of Mines and Energy and Ministry of Health and Social Welfare, Marathon EG Production Limited, Noble Energy and Atlantic Methanol Production Company.

\section{Publisher's Note}

Springer Nature remains neutral with regard to jurisdictional claims in published maps and institutional affiliations.

\section{Received: 6 March 2019 Accepted: 22 April 2019}

Published online: 29 April 2019

\section{References}

1. WHO. World malaria report 2018. Geneva: World Health Organization; 2019.

2. Clyde DF. Malaria in Tanzania. Oxford: Oxford University Press; 1967.

3. Drakeley CJ, Reyburn H, Malima R, Nkya WMMM, Lemnge MM, Lusingu JPA, et al. Altitude-dependent and -independent variations in Plasmodium falciparum prevalence in Northeastern Tanzania. J Infect Dis. 2005;191:1589-98.

4. Wongsrichanalai C, Barcus MJ, Muth S, Sutamihardja A, Wernsdorfer WH. A review of malaria diagnostic tools: microscopy and rapid diagnostic test (RDT). Am J Trop Med Hyg. 2007;77:119-27.

5. Mueller I, Zimmerman PA, Reeder JC. Plasmodium malariae and Plasmodium ovale — the "bashful" malaria parasites. Trends Parasitol. 2007;23:278-83.

6. Collins WE, Jeffery GM. Plasmodium malariae: parasite and disease. Clin Microbiol Rev. 2007;20:579-92.

7. Hendrickse RG, Adeniyi A, Edington GM, Glasgow EF, White RHR, Houba V. Quartan malarial nephrotic syndrome: collaborative clinicopathological study in Nigerian children. Lancet. 1972;299:1143-9.
8. Badiane AS, Diongue K, Diallo S, Ndongo AA, Diedhiou CK, Deme AB, et al. Acute kidney injury associated with Plasmodium malariae infection. Malar J. 2014:13:226.

9. da Silva GB, Pinto JR, Barros EJG, Farias GMN, Daher EDF. Kidney involvement in malaria: an update. Rev Inst Med Trop Sao Paulo. 2017;59:e53.

10. Woodford J, Collins KA, Odedra A, Wang C, Kyung Jl, Domingo GJ, et al. An experimental human blood stage model for studying Plasmodium malariae infection. J Infect Dis. 2019. https://doi.org/10.1093/infdis/jiz102.

11. Jongo SA, Shekalaghe SA, Preston Church LW, Ruben AJ, Schindler T, Zenklusen I, et al. Safety, immunogenicity, and protective efficacy against controlled human malaria infection of Plasmodium falciparum sporozoite vaccine in Tanzanian adults. Am J Trop Med Hyg. 2018;99:338-49.

12. Agnandji ST, Lell B, Soulanoudjingar SS, Fernandes JF, Abossolo BP, Conzelmann C, et al. First results of phase 3 trial of RTS, S/AS01 malaria vaccine in African children. N Engl J Med. 2011;365:1863-75.

13. Steiner-Monard V, Kamaka K, Karoui O, Roethlisberger S, Audran R, Daubenberger C, et al. The candidate blood-stage malaria vaccine p27a induces a robust humoral response in a fast track to the field phase 1 trial in exposed and nonexposed volunteers. Clin Infect Dis. 2019;68:466-74.

14. Shekalaghe S, Rutaihwa M, Billingsley PF, Chemba M, Daubenberger CA, James ER, et al. Controlled human malaria infection of Tanzanians by intradermal injection of aseptic, purified, cryopreserved Plasmodium falciparum sporozoites. Am J Trop Med Hyg. 2014;91:471-80.

15. Seder RA, Chang LJ, Enama ME, Zephir KL, Sarwar UN, Gordon IJ, et al. Protection against malaria by intravenous immunization with a nonreplicating sporozoite vaccine. Science. 2013;341:1359-65.

16. Ishizuka AS, Lyke KE, DeZure A, Berry AA, Richie TL, Mendoza FH, et al. Protection against malaria at 1 year and immune correlates following PfSPZ vaccination. Nat Med. 2016;22:614-23.

17. Lyke KE, Ishizuka AS, Berry AA, Chakravarty S, DeZure A, Enama ME, et al. Attenuated PFSPZ vaccine induces strain-transcending T cells and durable protection against heterologous controlled human malaria infection. Proc Natl Acad Sci USA. 2017;114:2711-6.

18. Sissoko MS, Healy SA, Katile A, Omaswa F, Zaidi I, Gabriel EE, et al. Safety and efficacy of PFSPZ vaccine against Plasmodium falciparum via direct venous inoculation in healthy malaria-exposed adults in Mali: a randomised, double-blind phase 1 trial. Lancet Infect Dis. 2017;17:498-509.

19. Epstein JE, Paolino KM, Richie TL, Sedegah M, Singer A, Ruben AJ, et al. Protection against Plasmodium falciparum malaria by PfSPZ vaccine. JCI Insight. 2017;2:e89154.

20. Jongo SA, Preston Church LW, Mtoro AT, Chakravarty S, Ruben AJ, Swanson PA, et al. Safety and differential antibody and T cell responses to PfSPZ vaccine by age in Tanzanian adults, adolescents, children and infants. Am J Trop Med Hyg. 2019. https://doi.org/10.4269/ajtmh.18-0835.

21. Kamau E, Alemayehu S, Feghali KC, Saunders D, Ockenhouse CF. Multiplex qPCR for detection and absolute quantification of malaria. PLOS ONE. 2013;8:e71539.

22. Reller ME, Chen WH, Dalton J, Lichay MA, Dumler JS. Multiplex 5' nuclease quantitative real-time PCR for clinical diagnosis of malaria and specieslevel identification and epidemiologic evaluation of malaria-causing parasites, including Plasmodium knowlesi. J Clin Microbiol. 2013;51:2931-8.

23. Han E-T, Watanabe R, Sattabongkot J, Khuntirat B, Sirichaisinthop J, Iriko $\mathrm{H}$, et al. Detection of four Plasmodium species by genus- and speciesspecific loop-mediated isothermal amplification for clinical diagnosis. J Clin Microbiol. 2007:45:2521-8.

24. Hofmann N, Mwingira F, Shekalaghe S, Robinson LJ, Mueller I, Felger I. Ultra-sensitive detection of Plasmodium falciparum by amplification of multi-copy subtelomeric targets. PLoS Med. 2015;12:e1001788.

25. Cnops L, Jacobs J, Van Esbroeck M. Validation of a four-primer real-time PCR as a diagnostic tool for single and mixed Plasmodium infections. Clin Microbiol Infect. 2011;17:1101-7.

26. Miller RH, Obuya CO, Wanja EW, Ogutu B, Waitumbi J, Luckhart S, et al. Characterization of Plasmodium ovale curtisi and P. ovale wallikeri in Western Kenya utilizing a novel species-specific real-time PCR assay. PLoS Negl Trop Dis. 2015;9:e0003469.

27. Schindler T, Robaina T, Sax J, Bieri JR, Mpina M, Gondwe L, et al. Molecular monitoring of the diversity of human pathogenic malaria species in blood donations on Bioko Island, Equatorial Guinea. Malar J. 2019;18:9. 